TRANSACTIONS OF THE

AMERICAN MATHEMATICAL SOCIETY

Volume 357, Number 12, Pages 4867-4883

S 0002-9947(05)04046-8

Article electronically published on July 19, 2005

\title{
AFFINE PSEUDO-PLANES AND CANCELLATION PROBLEM
}

\author{
KAYO MASUDA AND MASAYOSHI MIYANISHI
}

\begin{abstract}
We define affine pseudo-planes as one class of $\mathbb{Q}$-homology planes. It is shown that there exists an infinite-dimensional family of non-isomorphic affine pseudo-planes which become isomorphic to each other by taking products with the affine line $\mathbb{A}^{1}$. Moreover, we show that there exists an infinitedimensional family of the universal coverings of affine pseudo-planes with a cyclic group acting as the Galois group, which have the equivariant non-cancellation property. Our family contains the surfaces without the cancellation property, due to Danielewski-Fieseler and tom Dieck.
\end{abstract}

\section{INTRODUCTION}

Let $G$ be an algebraic group defined over the complex number field $\mathbb{C}$. We shall consider the following:

Equivariant Cancellation Problem. Let $X$ and $Y$ be smooth affine varieties with algebraic $G$-actions. If $X \times W$ is $G$-isomorphic to $Y \times W$ for a $G$-module $W$, is $X$ then $G$-isomorphic to $Y$ ?

If we forget the actions, the problem is simply called the Cancellation Problem. When $Y \cong \mathbb{A}^{2}$, the cancellation holds by the results of Miyanishi-Sugie [18] and Fujita [7. However, the Cancellation Problem for $Y \cong \mathbb{A}^{n}$ remains open if $n \geq 3$.

In the Equivariant Cancellation Problem, the intriguing case is when $Y$ is isomorphic to a $G$-module, i.e., an affine space with a linear $G$-action. In this case, it is known that the answer is negative. In fact, for a reductive algebraic group $G$, there exist affine spaces with non-linearizable $G$-actions which are realized as the total spaces of non-trivial algebraic $G$-vector bundles over $G$-modules (Schwarz [19, see also references in [10]). By Bass-Haboush [2], every $G$-vector bundle over a $G$-module is stably trivial, namely, it becomes isomorphic to a trivial $G$-vector bundle by adding a certain trivial $G$-vector bundle. Hence non-trivial $G$-vector bundles over $G$-modules, whose total spaces have non-linearizable $G$-actions, give rise to counterexamples to the Equivariant Cancellation Problem with $G$-modules $Y$ (cf. Masuda-Miyanishi [12]). All counterexamples to the Equivariant Cancellation Problem that we have so far for reductive algebraic groups $G$ and $G$-modules $Y$ are derived from non-trivial $G$-vector bundles over $G$-modules.

Next, consider the case where $Y$ is not isomorphic to a $G$-module nor an affine space without $G$-action. Then there are well-known counterexamples due to Daniel-

Received by the editors November 26, 2003.

2000 Mathematics Subject Classification. Primary 14R10; Secondary 14R20, 14R25, 14L30.

Key words and phrases. Equivariant Cancellation Problem, algebraic group action.

This work was supported by Grant-in-Aid for Scientific Research (C), JSPS.

(C)2005 American Mathematical Society Reverts to public domain 28 years from publication 4867 
ewski [4, Fieseler [6], tom Dieck [5], and Wilkens [22]. In tom Dieck [5], it is shown that the smooth affine surfaces $\widetilde{V}(d, r)$ in $\mathbb{A}^{3}$ defined by $x^{r} z+y^{d}=1$ for $r \geq 1$ and $d \geq 2$ have non-cancellation property; namely, $\widetilde{V}(d, r) \varsubsetneqq \widetilde{V}(d, s)$ for $r \neq s$, but $\widetilde{V}(d, r) \times \mathbb{A}^{1} \cong \widetilde{V}(d, s) \times \mathbb{A}^{1}$ for any $r$ and $s$. The surface $\widetilde{V}(d, r)$ has an action of the multiplicative group $G_{m}=\mathbb{C}^{*}$ and tom Dieck showed that the surfaces $\widetilde{V}(d, r)$ have in fact equivariant non-cancellation property. There exists an action of the additive group $G_{a}=\mathbb{C}^{+}$on $\widetilde{V}(d, r)$ as well, and hence there is an $\mathbb{A}^{1}$-fibration $\widetilde{\rho}: \widetilde{V}(d, r) \rightarrow \mathbb{A}^{1}=\operatorname{Spec} \mathbb{C}[x]$, which has a unique reducible fiber $\widetilde{\rho}^{-1}(0)$. We call a smooth affine surface $X$ an affine pseudo-plane if $X$ has an $\mathbb{A}^{1}$-fibration $\rho: X \rightarrow \mathbb{A}^{1}$ such that every fiber of $\rho$ is irreducible and there is only one multiple fiber. Then the surfaces $\widetilde{V}(d, r)$ are the universal coverings of affine pseudo-planes $V(d, r)$ with Galois group isomorphic to $\mathbb{Z} / d \mathbb{Z}$. The surfaces $V(d, r)$ constructed by tom Dieck for $r \geq 2$ are characterized as affine pseudo-planes with non-trivial $\mathbb{C}^{*}$-actions. See section 2 for definitions and relevant results.

In the present article, we observe various properties of affine pseudo-planes and their universal coverings. We shall show that affine pseudo-planes can be constructed from the Hirzebruch surfaces, as tom Dieck's surfaces $V(d, r)$ are constructed from the Hirzebruch surfaces. We also show that the universal coverings of affine pseudo-planes are isomorphic to the hypersurfaces in $\mathbb{A}^{3}$, and give their defining equations explicitly. Using the results on the properties of affine pseudo-planes and their universal coverings, we show that if $d \geq 2$, there exists an infinite-dimensional family of non-isomorphic smooth affine surfaces with actions of $G_{a} \times \mathbb{Z} / d \mathbb{Z}$, whose members are mutually equivariantly non-cancellative. The family consists of the universal coverings of affine pseudo-planes and includes the examples due to Danielewski-Fieseler and tom Dieck. By taking their quotients by $\mathbb{Z} / d \mathbb{Z}$, we also obtain an infinite-dimensional family of non-isomorphic affine pseudo-planes without cancellation property. In the last section, we show that there exist families of non-isomorphic affine $G$-varieties without equivariant cancellation property. The families are of infinite dimension and are derived from $G$-equivariant $\mathbb{A}^{1}$-fibrations and not $G$-vector bundles.

\section{Affine pseudo-planes with Unique $\mathbb{A}^{1}$-Fibrations}

An algebro-geometric characterization of the affine plane $\mathbb{A}^{2}$ is stated as follows: the affine plane $\mathbb{A}^{2}$ is an affine surface such that its coordinate ring $R$ is factorial, $R^{*}=\mathbb{C}^{*}$, and there exists an $\mathbb{A}^{1}$-fibration with base curve isomorphic to $\mathbb{A}^{1}$. There are many related results on smooth affine surfaces with $\mathbb{A}^{1}$-fibrations (cf. Miyanishi [14]). Here we recall the following.

Lemma 2.1. Let $X$ be a smooth affine surface with an $\mathbb{A}^{1}$-fibration $\rho: X \rightarrow C \cong$ $\mathbb{A}^{1}$. Suppose that every fiber of $\rho$ is irreducible. Then $\operatorname{Pic}(X) \cong \prod_{P \in C} \mathbb{Z} / d_{P} \mathbb{Z}$, where $d_{P}$ is the multiplicity of the fiber $\rho^{-1}(P)$. In particular, if there is only one multiple fiber $d F$ with the multiplicity $d$ and $F \cong \mathbb{A}^{1}$, then $\operatorname{Pic}(X) \cong \mathbb{Z} / d \mathbb{Z}$.

Affine surfaces satisfying the assumptions in Lemma 2.1 are $\mathbb{Q}$-homology planes, and there are many such surfaces. We define affine pseudo-plane as one class of such affine surfaces.

Definition 2.1. A smooth affine surface $X$ is an affine pseudo-plane if $X$ satisfies the following conditions. 
(1) $X$ has an $\mathbb{A}^{1}$-fibration $\rho: X \rightarrow C$, where $C \cong \mathbb{A}^{1}$.

(2) The $\mathbb{A}^{1}$-fibration $\rho$ has a unique multiple fiber $d F$ with multiplicity $d \geq 2$ and $F \cong \mathbb{A}^{1}$, and every other fiber is isomorphic to $\mathbb{A}^{1}$.

We say that $X$ has type $(d, n, r)$ if $X$ further satisfies the next condition:

(3) $X$ has a smooth compactification $(V, D)$ such that the boundary divisor $D=V-X$ has simple normal crossings and the dual graph of $D$ is as given in Figure 1 below, where $n \geq 1$ and $r \geq 1$. Furthermore, $\bar{F}$ is the closure of $F$ in $V$, and $S^{\prime}$ is the unique cross-section contained in $D$.

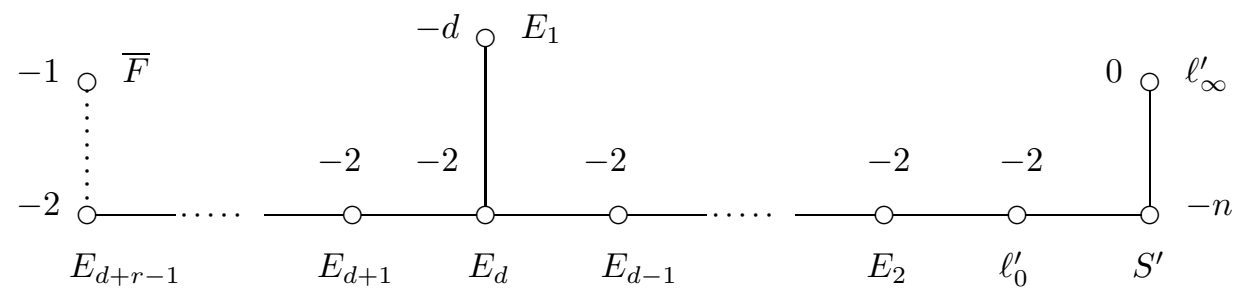

Figure 1

If $X$ has a smooth compactification $(V, D)$ with the dual graph as in Figure 1 and $\left(S^{\prime 2}\right)=-n$ for $n>1$, then we can make $\left(S^{\prime 2}\right)=-1$. In fact, choose a point $P$ on the fiber $\ell_{\infty}^{\prime}$ and blow up the point $P$ to obtain a $(-1)$ curve $E$. Then the proper transform $L$ of $\ell_{\infty}^{\prime}$ is a $(-1)$ curve. Contract $L$ to obtain the same figure as before with $\ell_{\infty}^{\prime}$ replaced by the image of $E$ and with $\left({S^{\prime}}^{2}\right)=-n+1$ if $P \neq S^{\prime} \cap \ell_{\infty}^{\prime}$, and $-n-1$ if $P=S^{\prime} \cap \ell_{\infty}^{\prime}$. This operation is called the elementary transformation with center $P$. After several elementary transformations, we obtain $\left({S^{\prime}}^{2}\right)=-1$. Meanwhile, we have to consider the case $\left({S^{\prime}}^{2}\right)<-1$ as well, e.g., in the proof of Theorem 2.3. We call an affine pseudo-plane of type $(d, 1, r)$ simply an affine pseudo-plane of type $(d, r)$.

Lemma 2.2. Let $X$ be an affine pseudo-plane of type $(d, r)$. Then $X$ is isomorphic to the complement of $M_{0} \cup C_{d}$ if $r<d$, and $M_{1} \cup C_{d}$ if $r \geq d$ in the Hirzebruch surface $\Sigma_{n}$ with $n=|r-d|$, where $M_{0}$ is the minimal section and where $C_{d}$ and $M_{1}$ are specified as follows. In the case $r<d, C_{d}$ is an irreducible member of the linear system $\left|M_{0}+d \ell_{0}\right|$ which meets $M_{0}$ in the point $M_{0} \cap \ell_{0}$ with multiplicity $r$, where $\ell_{0}$ is a fiber of the $\mathbb{P}^{1}$-fibration of $\Sigma_{n}$. In the case $r \geq d, M_{1}$ is a section of $\Sigma_{n}$ with $\left(M_{1}^{2}\right)=n$, and $C_{d}$ is an irreducible member of the linear system $\left|M_{1}+d \ell_{0}\right|$ which meets $M_{1}$ in the point $M_{1} \cap \ell_{0}$ with multiplicity $r$. In both cases, $\ell_{0} \cap X=\bar{F} \cap X$.

Proof. Contract $S^{\prime}, \ell_{0}^{\prime}, E_{2}, \ldots, E_{d}, E_{d+1}, \ldots, E_{d+r-1}$ in this order. Then the resulting surface is the Hirzebruch surface $\Sigma_{n}$ with $n=|r-d|$ and the image of $\ell_{\infty}^{\prime}$ provides $C_{d}$. The image of $E_{1}$ provides $M_{0}$ or $M_{1}$ according to whether $r-d<0$ or $r-d \geq 0$, while the image of $\bar{F}$ is the fiber $\ell_{0}$.

An affine pseudo-plane $X$ of type $(d, n, r)$ with $r \geq 2$ has the distinguished property as stated in the following theorem. An $\mathbb{A}^{1}$-fibration $\rho: X \rightarrow C \cong \mathbb{A}^{1}$ is called unique; if there is another $\mathbb{A}^{1}$-fibration $\sigma: X \rightarrow B \cong \mathbb{A}^{1}$, then $\sigma=\tau \circ \rho$ for an automorphism $\tau$ of $\mathbb{A}^{1}$. The next theorem follows from a theorem of Bertin [3], but we prefer to give a direct proof. 
Theorem 2.3. Let $X$ be an affine pseudo-plane of type $(d, n, r)$ with $r \geq 2$. Then $\rho$ is a unique $\mathbb{A}^{1}$-fibration on $X$.

Proof. Suppose that there exists another $\mathbb{A}^{1}$-fibration $\sigma: X \rightarrow B$ which is different from the fixed $\mathbb{A}^{1}$-fibration $\rho: X \rightarrow C$. Then $B \cong \mathbb{A}^{1}$ and every fiber of $\sigma$ is isomorphic to $\mathbb{A}^{1}$ if taken with the reduced structure. Let $M$ be a linear pencil on $V$ spanned by the closures of general fibers of $\sigma$, where the notations $V, D$, etc. are the same as in Definition 2.1. Then a general member of $M$ meets the curve $\ell_{\infty}^{\prime}$, for otherwise the $\mathbb{A}^{1}$-fibrations $\rho$ and $\sigma$ coincide with each other. Suppose that $M$ has no base points. Then the curve $\ell_{\infty}^{\prime}$ is a cross-section of $M$ and $S^{\prime}+\ell_{0}^{\prime}+E_{1}+$ $\cdots+E_{d+r-1}$ supports a reducible fiber of $M$. Then $r=d=1$. Since $d \geq 2$ by the hypothesis, this case does not take place. Hence $M$ has a base point, say $P$, on $\ell_{\infty}^{\prime}$. Let $Q:=\ell_{\infty}^{\prime} \cap S^{\prime}$. We consider two cases separately.

Case $P \neq Q$. Then $\ell_{\infty}^{\prime}+S^{\prime}+\ell_{0}^{\prime}+E_{1}+E_{2}+\cdots+E_{d+r-1}$ will support a reducible member $G_{0}$ of the pencil $M$. Let $s=(\bar{F} \cdot G)$, where $G$ is a general member of $M$. By comparing the intersection numbers of $G$ with two fibers of $\rho, \ell_{\infty}^{\prime}$ and the one containing $d \bar{F}$, it follows that $\left(\ell_{\infty}^{\prime} \cdot G\right)=d s$. Let $\mu$ be the multiplicity of $G$ at $P$, where $P$ is a one-place point of $G$. We have $d s \geq \mu$. Consider first the case $n=1$. The contraction of $S^{\prime}, \ell_{0}^{\prime}, E_{2}, \ldots, E_{d-1}$ makes $E_{d}$ a $(-1)$ curve meeting three components $\ell_{\infty}^{\prime}, E_{1}, E_{d+1}$, and this is impossible. So, suppose $n \geq 2$. The elimination of the base points of $M$ will be achieved by blowing up the point $P$ and its infinitely near points. After the elimination of the base points of $M$, the proper transform $\widetilde{M}$ gives rise to a $\mathbb{P}^{1}$-fibration, and the proper transform of $\ell_{\infty}^{\prime}$ is a unique $(-1)$ component. If $g s>\mu$, then the point $P$ and its infinitely near point of the first order lying on $\ell_{\infty}^{\prime}$ are blown up. Hence the proper transform of $\ell_{\infty}^{\prime}$ is not a $(-1)$ curve. This implies that $d s=\mu$. Let $E$ be the exceptional curve arising from the blowing-up of $P$ and let $M^{\prime}$ be the proper transform of $M$. Then $E$ is contained in the member $G_{0}^{\prime}$ of $M^{\prime}$ corresponding to $G_{0}$ of $M$. In fact, we otherwise have $d s=1$, which is impossible because $d \geq 2$. Now contract $\ell_{\infty}^{\prime}$ and take the image of $E$ instead of $\ell_{\infty}^{\prime}$. Then we have the same dual graph as Figure 1 with $\left({S^{\prime}}^{2}\right)=-(n-1)$. By repeating this argument, we reach a contradiction.

Case $P=Q$. As above, let $G_{0}$ be a reducible member of $M$ containing $S^{\prime}+\ell_{0}^{\prime}+$ $E_{1}+E_{2}+\cdots+E_{d+r-1}$. If $\ell_{\infty}^{\prime}$ is not contained in $G_{0}$, the elimination of the base points of $M$, which is achieved by blowing up the point $P=Q$ and its infinitely near points, yields a $\mathbb{P}^{1}$-fibration in which the fiber corresponding to $G_{0}$ is a reducible fiber not containing any $(-1)$ curve. This is a contradiction. Hence $\ell_{\infty}^{\prime}$ is contained in $G_{0}$. So, $G_{0}$ is supported by $S^{\prime}+\ell_{0}^{\prime}+E_{1}+E_{2}+\cdots+E_{d+r-1}+\ell_{\infty}^{\prime}$. Now apply the elementary transformation with center $P$. Then we obtain the same dual graph as Figure 1, where $\left({S^{\prime}}^{2}\right)=-(n+1)$ and $\ell_{\infty}^{\prime}$ is replaced by the image of $E$. After repeating the elementary transformations several times, we are reduced to the case where $P \neq Q$. So, we reach a contradiction in the present case as well.

Since the existence of an $\mathbb{A}^{1}$-fibration with affine base is equivalent to the existence of an action of the additive group $G_{a}$, it follows from Theorem 2.3 that there is an essentially unique $G_{a}$-action on an affine pseudo-plane of type $(d, n, r)$ for $r \geq 2$. On the other hand, an affine pseudo-plane of type $(d, n, 1)$ has two algebraically independent $G_{a}$-actions, namely, it has trivial Makar-Limanov invariant (cf. [11]). This is a consequence of a more general result in Gurjar-Miyanishi [9, Theorem 3.1] which is stated below. We only note that the boundary divisor $D$ in the case of 
type $(d, n, 1)$ is a linear chain for the normal compactification in Definition 2.1 and that $\pi_{1, \infty}(X)$ is then a finite cyclic group of order $d^{2}$.

Theorem 2.4. Let $X$ be a smooth affine surface. Then the Makar-Linamov invariant $\operatorname{ML}(X)$ is trivial if and only if $X$ has a minimal normal compactification $V$ such that the dual graph of $D:=V-X$ is a linear chain of rational curves and $\pi_{1, \infty}(X)$ is a finite group.

Lemma 2.2 gives rise to a construction of affine pseudo-planes from the Hirzebruch surfaces. We denote by $X(d, r)$ an affine pseudo-plane of type $(d, r)$ constructed from the Hirzebruch surface as in Lemma 2.2. Some partial cases of affine pseudo-planes were observed in tom Dieck [5] as examples of affine surfaces without cancellation property. We shall recall and generalize a little bit his construction. Write $\Sigma_{n}=\operatorname{Proj}\left(\mathcal{O}_{\mathbb{P}^{1}}(-n) \oplus \mathcal{O}_{\mathbb{P}^{1}}\right)$ as the quotient of $\left(\mathbb{A}^{2} \backslash\{0\}\right) \times \mathbb{P}^{1}$ under the relation

$$
\left(z_{0}, z_{1}\right),\left[w_{0}, w_{1}\right] \sim\left(\nu z_{0}, \nu z_{1}\right),\left[\nu^{n} w_{0}, w_{1}\right]
$$

for $\nu \in G_{m}=\mathbb{C}^{*}$. The projection $\left\{\left(z_{0}, z_{1}\right),\left[w_{0}, w_{1}\right]\right\} \mapsto\left[z_{0}, z_{1}\right]$ induces a $\mathbb{P}^{1}$ fibration $p_{n}: \Sigma_{n} \rightarrow \mathbb{P}^{1}$. In the above definition by quotient and in what follows, the integer $n$ could be negative. If $n \geq 0$, the curve $w_{0}=0$ (resp. $\left.w_{1}=0\right)$ is a section $M_{1}$ of $p_{n}$ with $\left(M_{1}^{2}\right)=n$ (resp. the minimal section $M_{0}$ with $\left.\left(M_{0}{ }^{2}\right)=-n\right)$. Meanwhile, if $n<0$, then the curve $w_{0}=0$ (resp. $w_{1}=0$ ) is the minimal section $M_{0}$ (resp. a section $M_{1}$ with $\left.\left(M_{1}^{2}\right)=|n|\right)$ of $\Sigma_{|n|}$. Let $d \geq 2$ and $r=d+n \geq 1$. With the notations of Lemma 2.2, we assume that the fiber $\ell_{0}$ is defined by $z_{0}=0$. Let $w=w_{0} / w_{1}$. Then $\left\{z_{0} / z_{1}, w / z_{1}^{n}\right\}$ is a system of local coordinates at the point $M_{1} \cap \ell_{0}$ (resp. $M_{0} \cap \ell_{0}$ ) if $n \geq 0$ (resp. $n<0$ ). Let $\Lambda$ be a linear subsystem of $\left|M_{1}+d \ell_{0}\right|$ if $n \geq 0$ (resp. $\left|M_{0}+d \ell_{0}\right|$ if $n<0$ ), consisting of members which meet the curve $M_{1}$ (resp. $M_{0}$ ) at the point $M_{1} \cap \ell_{0}$ (resp. $M_{0} \cap \ell_{0}$ ) with multiplicity $r$ if $n \geq 0$ (resp. $n<0$ ). Then any member of $\Lambda$ is defined by an equation

$$
\frac{w}{z_{1}^{n}}\left\{a_{0}+a_{1}\left(\frac{z_{0}}{z_{1}}\right)+\cdots+a_{d-1}\left(\frac{z_{0}}{z_{1}}\right)^{d-1}+a_{d}\left(\frac{z_{0}}{z_{1}}\right)^{d}\right\}+a_{d+1}\left(\frac{z_{0}}{z_{1}}\right)^{r}=0
$$

or equivalently by

$$
w_{0}\left(a_{0} z_{1}^{d}+a_{1} z_{0} z_{1}^{d-1}+\cdots+a_{d-1} z_{0}^{d-1} z_{1}+a_{d} z_{0}^{d}\right)+a_{d+1} z_{0}^{r} w_{1}=0
$$

for $\left(a_{0}, a_{1}, \ldots, a_{d+1}\right) \in \mathbb{P}^{d+1}$. In fact, it is readily computed that $\operatorname{dim} \Lambda=d+1$. So, the curve $C_{d}$ is defined by such an equation with $a_{0} \neq 0$ and $a_{d+1} \neq 0$. Hence it follows that

$$
X(d, r)=\Sigma_{|n|} \backslash\left(\left\{w_{0}=0\right\} \cup C_{d}\right)
$$

where $n=r-d$ and $C_{d}$ is the curve defined by (1) with $a_{0} \neq 0$ and $a_{d+1} \neq 0$. We shall verify the following result.

Lemma 2.5. Let $r \geq 2$ and let $X=X(d, r)$ be an affine pseudo-plane defined as above. Let $\sigma: G_{m} \times X \rightarrow X$ be a non-trivial action of the algebraic torus $G_{m}=\mathbb{C}^{*}$. Then the following assertions hold true:

(1) The action $\sigma$ induces an action $\sigma: G_{m} \times \Sigma_{|n|} \rightarrow \Sigma_{|n|}$ such that ${ }^{\sigma(\mu)} M_{i} \subseteq M_{i}$ for $i=0,1,{ }^{\sigma(\mu)} C_{d} \subseteq C_{d}$ and ${ }^{\sigma(\mu)} \ell_{0} \sim \ell_{0}$, where ${ }^{\sigma(\mu)} M_{i}$ denotes the image of $M_{i}$ under the action of $\mu \in \mathbb{C}^{*}$, etc.

(2) The curve $C_{d}$ is defined by an equation

$$
z_{1}^{d} w_{0}+a z_{0}^{r} w_{1}=0 \quad \text { for } \quad a \in \mathbb{C}^{*} .
$$


Proof. (1) We prove only the case $n=r-d \geq 0$. The proof of the case $n<0$ is done in the same manner. Let $\rho: X \rightarrow C \cong \mathbb{A}^{1}$ be the unique $\mathbb{A}^{1}$-fibration (cf. Theorem 2.3). Then the fibers of $\rho$ are permuted by the action $\sigma$. Hence $\sigma$ extends to the cross-section $S^{\prime}$ and sends $S^{\prime}$ into itself. Let $W$ be a $G_{m}$-equivariant smooth normal compactification of $X$ whose existence is guaranteed by 21. We may assume that $W \backslash X$ contains the cross-section $S^{\prime}$. Let $F_{0}$ and $F_{\infty}$ be two fibers of the $\mathbb{P}^{1}$-fibration $p: W \rightarrow \mathbb{P}^{1}$ whose supports partly or totally lie outside of $X$, where $F_{0}$ contains the multiple fiber of $\rho$. We may assume that all $(-1)$ components of $F_{0}$ and $F_{\infty}$ are fixed componentwise under the action $\sigma$. Then we may assume that $F_{\infty}$ is irreducible and $F_{0}$ minus the component $\bar{F}$ contains no $(-1)$ components, where $\bar{F} \cap X$ gives rise to the multiple fiber of $\rho$. Then we may assume that $W \backslash X$ has the dual graph as in Definition 2.1. So, the action $\sigma$ induces a $G_{m}$-action on $\Sigma_{n}$ such that ${ }^{\sigma(\mu)}\left(M_{1}\right) \subseteq M_{1},{ }^{\sigma(\mu)}\left(C_{d}\right) \subseteq C_{d}$ and ${ }^{\sigma(\mu)}\left(\ell_{0}\right) \subseteq \ell_{0}$ because $M_{1}, C_{d}, \ell_{0}$ are the images on $\Sigma_{n}$ of the components $E_{1}, \ell_{\infty}^{\prime}, \bar{F}$, respectively. The minimal section $M_{0}$ is stable under the $\sigma$-action because the minimal section is unique on $\Sigma_{n}$.

(2) The $G_{m}$-action $\sigma$ on $\Sigma_{|n|}$ is given as follows in terms of the coordinates:

$$
\mu \cdot\left(\left(z_{0}, z_{1}\right),\left[w_{0}, w_{1}\right]\right)=\left(\left(\mu^{\alpha} z_{0}, \mu^{\beta} z_{1}\right),\left[\mu^{\gamma} w_{0}, \mu^{\delta} w_{1}\right]\right)
$$

for $\mu \in \mathbb{C}^{*}$. Since $C_{d}$ is stable under the $\sigma$-action, the defining equation (1) must be semi-invariant. Note that $a_{0} a_{d+1} \neq 0$ because $C_{d}$ is irreducible. Hence we obtain $\alpha r+\delta=\beta d+\gamma$. Suppose that $\left(a_{1}, \ldots, a_{d}\right) \neq(0, \ldots, 0)$. Then we have an additional relation $\alpha i+\beta(d-i)+\gamma=\beta d+\gamma$ for some $1 \leq i \leq d$. The last relation implies $\alpha=\beta$. So, the first relation gives $\gamma=\alpha n+\delta$. Then we have

$$
\begin{aligned}
\mu \cdot\left(\left(z_{0}, z_{1}\right),\left[w_{0}, w_{1}\right]\right) & =\left(\left(\mu^{\alpha} z_{0}, \mu^{\beta} z_{1}\right),\left[\mu^{\gamma} w_{0}, \mu^{\delta} w_{1}\right]\right) \\
& =\left(\left(\mu^{\alpha} z_{0}, \mu^{\beta} z_{1}\right),\left[\mu^{\alpha n+\delta} w_{0}, \mu^{\delta} w_{1}\right]\right) \\
& \sim\left(\left(z_{0}, z_{1}\right),\left[\mu^{\delta} w_{0}, \mu^{\delta} w_{1}\right]\right) \\
& =\left(\left(z_{0}, z_{1}\right),\left[w_{0}, w_{1}\right]\right) .
\end{aligned}
$$

Hence the $\sigma$-action is trivial. This proves the second assertion.

Let $V(d, r)$ be the affine pseudo-plane defined by

$$
V(d, r)=\Sigma_{|n|} \backslash\left(\left\{w_{0}=0\right\} \cup\left\{z_{1}^{d} w_{0}+z_{0}^{r} w_{1}=0\right\}\right)
$$

where $n=r-d$. Then there exists a $G_{m}$-action on $V(d, r)$ defined by

$$
\mu \cdot\left(\left(z_{0}, z_{1}\right),\left[w_{0}, w_{1}\right]\right)=\left(\left(\mu z_{0}, z_{1}\right),\left[w_{0}, \mu^{-r} w_{1}\right]\right)
$$

for $\mu \in \mathbb{C}^{*}$. For $r \geq 2$, one can show that any $G_{m}$-action on $V(d, r)$ is reduced to the $G_{m}$-action specified as above. In fact, with the notation in the proof of Lemma $2.5(2)$

$$
\begin{aligned}
\mu \cdot\left(\left(z_{0}, z_{1}\right),\left[w_{0}, w_{1}\right]\right) & =\left(\left(\mu^{\alpha} z_{0}, \mu^{\beta} z_{1}\right),\left[\mu^{\gamma} w_{0}, \mu^{\delta} w_{1}\right]\right) \\
& =\left(\left(\mu^{\alpha} z_{0}, \mu^{\beta} z_{1}\right),\left[\mu^{\alpha r-\beta d+\delta} w_{0}, \mu^{\delta} w_{1}\right]\right) \\
& =\left(\left(\mu^{\alpha} z_{0}, \mu^{\beta} z_{1}\right),\left[\mu^{\beta n+(\alpha-\beta) r+\delta} w_{0}, \mu^{\delta} w_{1}\right]\right) \\
& \sim\left(\left(\mu^{\alpha-\beta} z_{0}, z_{1}\right),\left[\mu^{r(\alpha-\beta)} w_{0}, w_{1}\right]\right) \\
& =\left(\left(\mu^{\alpha-\beta} z_{0}, z_{1}\right),\left[w_{0}, \mu^{-r(\alpha-\beta)} w_{1}\right]\right) .
\end{aligned}
$$

We shall consider the universal covering $\widetilde{X}(d, r)$ of an affine pseudo-plane $X(d, r)$. 
Lemma 2.6. The following assertions hold true:

(1) The universal covering $\widetilde{X}(d, r)$ is isomorphic to an affine hypersurface in $\mathbb{A}^{3}=\operatorname{Spec} \mathbb{C}[x, y, z]$ defined by an equation

$$
x^{r} z+\left(y^{d}+a_{1} x y^{d-1}+\cdots+a_{d-1} x^{d-1} y+a_{d} x^{d}\right)=1 .
$$

The Galois group of the covering $\widetilde{X}(d, r) \rightarrow X(d, r)$ is a cyclic group $H(d):=\mathbb{Z} / d \mathbb{Z}$ of order $d$ and acts as

$$
\lambda \cdot(x, y, z)=\left(\lambda x, \lambda y, \lambda^{-r} z\right)
$$

for $\lambda \in H(d)$.

(2) The projection $(x, y, z) \mapsto x$ induces an $\mathbb{A}^{1}$-fibration $\widetilde{\rho}: \widetilde{X}(d, r) \rightarrow \mathbb{A}^{1}$ such that every fiber except for $\widetilde{\rho}^{-1}(0)$ is smooth and the fiber $\widetilde{\rho}^{-1}(0)$ consists of $d$ copies of $\mathbb{A}^{1}$ which are reduced.

(3) There is a $G_{a}$-action on $\widetilde{X}(d, r)$ defined by

$$
\begin{aligned}
& c \cdot(x, y, z) \\
& \quad=\left(x, y+c x^{r}, z-x^{-r}\left\{\left(\left(y+c x^{r}\right)^{d}+a_{1} x\left(y+c x^{r}\right)^{d-1}+\cdots+a_{d} x^{d}\right)\right.\right. \\
& \left.\left.-\left(y^{d}+a_{1} x y^{d-1}+\cdots+a_{d-1} x^{d-1} y+a_{d} x^{d}\right)\right\}\right),
\end{aligned}
$$

where $c \in G_{a}=\mathbb{C}$.

(4) The $\mathbb{A}^{1}$-fibration $\tilde{\rho}: \tilde{X}(d, r) \rightarrow \mathbb{A}^{1}$ is unique for $r \geq 2$.

(5) Let $\omega$ be a d-th root of unity. Then there exist uniquely determined polynomials $p_{\omega}(x), q_{\omega}(x) \in \mathbb{C}[x]$ satisfying the following conditions:

(i) $\operatorname{deg} p_{\omega}(x) \leq r-1$.

(ii) $p_{\omega}(0)=\omega$.

(iii) $x^{r} q_{\omega}(x)+p_{\omega}(x)^{d}+a_{1} x p_{\omega}(x)^{d-1}+\cdots+a_{d-1} x^{d-1} p_{\omega}(x)+a_{d} x^{d}=1$.

(iv) $p_{\lambda \omega}(\lambda x)=\lambda p_{\omega}(x), q_{\lambda \omega}(\lambda x)=\lambda^{-r} q_{\omega}(x)$ for any $d$-th root $\lambda$ of unity.

By making use of these polynomials, we define the morphism

$$
\varphi_{\omega}: \mathbb{A}^{2} \cong \mathbb{A}^{1} \times G_{a} \rightarrow \widetilde{X}(d, r), \quad(x, c) \mapsto c \cdot\left(x, p_{\omega}(x), q_{\omega}(x)\right)
$$

which is an open immersion onto an open set $U_{\omega}$ which is the complement of $\coprod_{\lambda \neq \omega} G_{a} \cdot(0, \lambda, 0)$. The inverse morphism $\varphi_{\omega}^{-1}$ on $U_{\omega}$ is defined by

$$
(x, y, z) \mapsto \begin{cases}\left(x, \frac{y-p_{\omega}(x)}{x^{r}}\right) & \text { if } x \neq 0, \\ \left(0, \frac{-z+q_{\omega}(0)}{d \omega^{-1}}\right) & \text { if } x=0 .\end{cases}
$$

(6) $\widetilde{X}(d, r)$ is obtained by glueing together the d-copies of the affine plane $\mathbb{A}^{2}$ by the transition functions

$$
\begin{aligned}
g_{\lambda \omega}:=\varphi_{\lambda}^{-1} \circ \varphi_{\omega}: \mathbb{A}_{*}^{1} \times \mathbb{A}^{1} & \rightarrow \mathbb{A}_{*}^{1} \times \mathbb{A}^{1} \\
(x, c) & \mapsto\left(x, c+\frac{p_{\omega}(x)-p_{\lambda}(x)}{x^{r}}\right),
\end{aligned}
$$

where $\omega, \lambda \in H(d)$ and $\mathbb{A}_{*}^{1}=\mathbb{A}^{1}-\{0\}$.

(7) The Galois group $H(d)$ acts as

$$
\lambda \cdot \varphi_{\omega}(x, c)=\varphi_{\lambda \omega}\left(\lambda x, \lambda^{1-r} c\right) .
$$


Proof. (1) Recall that $X(d, r)$ is the complement in $\Sigma_{|n|}$ of the curves $C_{d}$ defined by the equation (1) with $a_{0} \neq 0$ and $a_{d+1} \neq 0$, and the curve $w_{0}=0$ which is $M_{1}$ if $r-d \geq 0$ (resp. $M_{0}$ if $r-d<0$ ), where $n=r-d$. Since $w_{0} \neq 0$, we can normalize to $w_{0}=1$. We can then normalize

$$
w_{0}\left(a_{0} z_{1}^{d}+a_{1} z_{0} z_{1}^{d-1}+\cdots+a_{d-1} z_{0}^{d-1} z_{1}+a_{d} z_{0}^{d}\right)+a_{d+1} z_{0}^{r} w_{1} \neq 0
$$

to the relation

$$
z_{0}^{r} w_{1}+\left(a_{0} z_{1}^{d}+a_{1} z_{0} z_{1}^{d-1}+\cdots+a_{d-1} z_{0}^{d-1} z_{1}+a_{d} z_{0}^{d}\right)=1,
$$

where $a_{0} \neq 0$. This normalization comes from the defining equivalence relation

$$
\left(z_{0}, z_{1}\right),\left[w_{0}, w_{1}\right] \sim\left(\nu z_{0}, \nu z_{1}\right),\left[\nu^{n} w_{0}, w_{1}\right] .
$$

We may assume that $a_{0}=1$. The equivalence relation requires that the points $\left(\lambda z_{0}, \lambda z_{1}, \lambda^{-n} w_{1}\right)$ for $\lambda \in H(d)$ should be identified together, where we note that $n=r-d$. Hence the assertion follows. Note that $\widetilde{X}(d, r)$ is simply connected.

(3) Let $\delta$ be a derivation on the coordinate ring $\Gamma(\widetilde{X}(d, r))$ defined by

$$
\begin{aligned}
& \delta(x)=0, \quad \delta(y)=x^{r}, \\
& \delta(z)=-\left(d y^{d-1}+(d-1) a_{1} x y^{d-2}+\cdots+a_{d-1} x^{d-1}\right) .
\end{aligned}
$$

Then $\delta$ is locally nilpotent. Hence it defines a $G_{a}$-action on $\widetilde{X}(d, r)$ by

$$
c \cdot a=\sum_{n=0}^{\infty} \frac{c^{n}}{n !} \delta^{n}(a), \quad \text { for } c \in G_{a}, a \in \Gamma(\widetilde{X}(d, r)),
$$

which is as specified as in the assertion. One easily verifies that $\operatorname{Ker} \delta=\mathbb{C}[x]$ and the inclusion $\operatorname{Ker} \delta \hookrightarrow \Gamma(\widetilde{X}(d, r))$ induces an $\mathbb{A}^{1}$-fibration $\tilde{\rho}: \widetilde{X}(d, r) \rightarrow \mathbb{A}^{1}$.

(4) We consider the smooth compactification $(V, D)$ as given in Definition 2.1. Then we have a linear equivalence

$$
\ell_{\infty}^{\prime} \sim \ell_{0}^{\prime}+E_{1}+2 E_{2}+\cdots+(d-1) E_{d-1}+d E_{d}+d\left(E_{d+1}+\cdots+E_{d+r-1}+\bar{F}\right),
$$

which is written as follows:

$$
\begin{aligned}
\ell_{\infty}^{\prime} & +(d-1)\left(\ell_{0}^{\prime}+E_{1}\right)+(d-2) E_{2}+\cdots+E_{d-1} \\
& \sim d\left\{\ell_{0}^{\prime}+E_{1}+E_{2}+\cdots+E_{d-1}+E_{d}+E_{d+1}+\cdots+E_{d+r-1}+\bar{F}\right\} .
\end{aligned}
$$

Let $q: \widetilde{V} \rightarrow V$ be a $d$-ple cyclic covering which ramifies totally over the branch locus $\ell_{\infty}^{\prime}+\ell_{0}^{\prime}+E_{1}+E_{2}+\cdots+E_{d-1}$. Then $\widetilde{V}$ has cyclic quoteint singularities over the intersection points $\ell_{0}^{\prime} \cap E_{2}, E_{2} \cap E_{3}, \ldots, E_{d-2} \cap E_{d-1}$. The minimal resolution of these singularities will only insert linear chains of exceptional curves in between the proper transforms of the intersecting curves. Meanwhile, the inverse image $\widetilde{E}_{d}:=q^{-1}\left(E_{d}\right)$ of the curve $E_{d}$ remains irreducible on $\widetilde{V}$ and the linear branch $E_{d+1}+\cdots+E_{d+r-1}+\bar{F}$ splits into a disjoint union of $d$ linear branches $\widetilde{E}_{d+1}^{(j)}+$ $\cdots+\widetilde{E}_{d+r-1}^{(j)}+\widetilde{\bar{F}}^{(j)}(1 \leq j \leq d)$. Each of the curves $\widetilde{E}_{d+1}^{(j)}(1 \leq j \leq d)$ meets the curve $\widetilde{E}_{d}$ transversally in one point. Furthermore, $q^{-1}(X(d, r))$ is the universal covering space $\widetilde{X}(d, r)$. By the above observations, one knows that the boundary divisor of $\widetilde{X}(d, r)$ embedded minimally in a smooth projective surface, which is obtained from $\widetilde{V}$ by resolving minimally the above cyclic quotient singularities and contracting $(-1)$ curves and consecutively contractible curves resulting from the inverse image of $q^{-1}\left(\ell_{\infty}^{\prime}+S^{\prime}+\ell_{0}^{\prime}+E_{1}+\cdots+E_{d-1}\right)$, is not a linear chain, for 
$\widetilde{E}_{d}+\sum_{j=1}^{d}\left(\widetilde{E}_{d+1}^{(j)}+\cdots+\widetilde{E}_{d+r-1}^{(j)}\right)$ cannot become a part of a linear chain if $d \geq 2$ and $r \geq 2$. So, we are done by a theorem of Bertin [3].

(5) Write $p_{\omega}(x)=\omega+c_{1}(\omega) x+\cdots+c_{r-1}(\omega) x^{r-1}$, where the coefficients are to be determined by the relation

$$
x^{r} q_{\omega}(x)+p_{\omega}(x)^{d}+a_{1} x p_{\omega}(x)^{d-1}+\cdots+a_{d-1} x^{d-1} p_{\omega}(x)+a_{d} x^{d}=1
$$

which is obtained from equation (2) above by substituting $p_{\omega}(x), q_{\omega}(x)$ for $y, z$. By condition (i), it is easy to see that $p_{\omega}(x)$ is uniquely determined. Namely the coefficients $c_{1}(\omega), \ldots, c_{r-1}(\omega)$ are uniquely determined by putting the coefficients of the terms $x^{i}(1 \leq i \leq r-1)$ to be zero in the left-hand side of equation (3) above. Then $q_{\omega}(x)$ is uniquely determined as well. By multiplying $\lambda^{d}=1$ to the relation (3), we obtain

$$
\begin{aligned}
& (\lambda x)^{r} \lambda^{-r} q_{\omega}\left(\lambda^{-1}(\lambda x)\right) \\
& \quad+\left(\lambda p_{\omega}\left(\lambda^{-1}(\lambda x)\right)\right)^{d}+a_{1}(\lambda x)\left(\lambda p_{\omega}\left(\lambda^{-1}(\lambda x)\right)\right)^{d-1}+\cdots+a_{d}(\lambda x)^{d}=1 .
\end{aligned}
$$

Replace $\lambda x$ by $x$ in the above relation. Then the uniqueness of the polynomials $p_{\lambda \omega}(x), q_{\lambda \omega}(x)$ imply that $p_{\lambda \omega}(x)=\lambda p_{\omega}\left(\lambda^{-1} x\right)$ and $q_{\lambda \omega}(x)=\lambda^{-r} q_{\omega}\left(\lambda^{-1} x\right)$. Now replace $x$ by $\lambda x$. Then we obtain the relation (iv). Note that $\varphi_{\omega}: \mathbb{A}^{2} \rightarrow U_{\omega}$ is injective and $U_{\omega} \cong \mathbb{A}^{2}$. Hence $\varphi_{\omega}$ is an isomorphism by [1]. The $\varphi_{\omega}(\omega \in H(d))$ are bundle charts of $\widetilde{X}(d, r)$ and $\widetilde{X}(d, r)$ is obtained by glueing $d$-copies $\{\omega\} \times \mathbb{A}^{2}$ $(\omega \in H(d))$ under the identification

$$
(\omega, x, c) \sim\left(\lambda, x, c+\frac{p_{\omega}(x)-p_{\lambda}(x)}{x^{r}}\right)
$$

for $\omega, \lambda \in H(d)$ and $(x, c) \in \mathbb{A}_{*}^{1} \times \mathbb{A}^{1}$. The other assertions are verified in a straightforward manner.

We say that a homogeneous polynomial $y^{d}+a_{1} x y^{d-1}+\cdots+a_{d} x^{d}$ with the coefficient of the $y^{d}$-term equal to 1 is monic. Let $\widetilde{X}(d, r)$ and $\widetilde{X^{\prime}}(d, s)$ be the affine hypersurfaces in $\mathbb{A}^{3}$ defined by $x^{r} z+f(x, y)=1$ and $x^{s} z+h(x, y)=1$, respectively, where $f(x, y)$ and $h(x, y)$ are monic homogeneous polynomials of degree $d$. If $r \neq s$, then $\widetilde{X}(d, r) \neq \widetilde{X^{\prime}}(d, s)$ since $\pi_{1, \infty}(\widetilde{X}(d, r)) \neq \pi_{1, \infty}\left(\widetilde{X^{\prime}}(d, s)\right)$. Concerning the isomorphism classes of the hypersurfaces $\widetilde{X}(d, r)$, we have the following result. Note that we may assume that $f(x, y)$ and $h(x, y)$ are of the form $y^{d}+a_{2} x^{2} y^{d-2}+\cdots+a_{d} x^{d}$ by changing the coordinates.

Lemma 2.7. Let $d \geq 2$ and $r>d$. Let $\widetilde{X}_{1}(d, r)$ and $\widetilde{X}_{2}(d, r)$ be the hypersurfaces defined by the equations $x^{r} z+y^{d}+a_{2} x^{2} y^{d-2}+\cdots+a_{d} x^{d}=1$ and $x^{r} z+y^{d}+b_{2} x^{2} y^{d-2}+$ $\cdots+b_{d} x^{d}=1$, respectively. Then there is an isomorphism $\widetilde{X}_{1}(d, r) \cong \widetilde{X}_{2}(d, r)$ if and only if

$$
a_{i}=\mu^{i} b_{i}, \quad \text { for } \quad \mu \in \mathbb{C}^{*}, \quad 2 \leq i \leq d .
$$

Proof. Let $f: \widetilde{X}_{1}(d, r) \rightarrow \widetilde{X}_{2}(d, r)$ be an isomorphism and let $\varphi$ be the induced isomorphism of the coordinate rings. Note that $f$ preserves the unique $\mathbb{A}^{1}$-fibrations of $\widetilde{X}_{1}(d, r)$ and $\widetilde{X}_{2}(d, r)$ as well as the reduced reducible fibers. Hence $f$ induces an automorphism of $\mathbb{C}[x]$ and $\varphi(x)=\mu x$ with $\mu \in \mathbb{C}^{*}$. Then $\varphi$ induces an automorphism of the polynomial ring $\mathbb{C}\left[x, x^{-1}\right][y]$. So, one can write $\varphi(y)=u x^{e} y+F(x)$ with $u \in \mathbb{C}^{*}, e \in \mathbb{Z}$ and $F(x) \in \mathbb{C}\left[x, x^{-1}\right]$. Since $\varphi$ is an isomorphism of the coordinate rings, it follows that $e \geq 0$ and $F \in \mathbb{C}[x]$. Furthermore, since $f$ maps 
isomorphically the unique reducible fiber of $\widetilde{X}_{1}(d, r)$ to the unique reducible fiber of $\widetilde{X}_{2}(d, r)$, it follows that $e=0, F(0)=0$ and $u^{d}=1$. Since

$$
\begin{aligned}
z & =x^{-r}\left\{1-\left(y^{d}+b_{2} x^{2} y^{d-2}+\cdots+b_{d} x^{d}\right)\right\} \\
& \in \Gamma\left(\widetilde{X}_{2}(d, r)\right) \otimes \mathbb{C}\left[x, x^{-1}\right]=\mathbb{C}\left[x, x^{-1}\right][y],
\end{aligned}
$$

it follows that $\varphi(z)=\mu^{-r} x^{-r}\left\{1-\left(\varphi(y)^{d}+b_{2}(\mu x)^{2} \varphi(y)^{d-2}+\cdots+b_{d}(\mu x)^{d}\right)\right\}$ in $\Gamma\left(\widetilde{X}_{1}(d, r)\right) \otimes \mathbb{C}\left[x, x^{-1}\right]=\mathbb{C}\left[x, x^{-1}\right][y]$. While, $\varphi$ is an isomorphism from $\Gamma\left(\widetilde{X}_{2}(d, r)\right)$ to $\Gamma\left(\widetilde{X}_{1}(d, r)\right), \varphi(z)$ is written in a form $\sum_{k>0}^{N} \varphi_{k}(x, y) z^{k}$, where $\varphi_{k}(x, y)=$ $\sum_{0 \leq j<d} \phi_{k j}(x) y^{j}$ for $\phi_{k j}(x) \in \mathbb{C}[x]$. Hence we have $\varphi(z)=\mu^{-r} z+\varphi_{0}(x, y)$, and

$$
(\varphi(y))^{d}+b_{2} \mu^{2} x^{2}(\varphi(y))^{d-2}+\cdots+b_{d} \mu^{d} x^{d}-1
$$

coincides with

$$
y^{d}+a_{2} x^{2} y^{d-2}+\cdots+a_{d} x^{d}-1
$$

modulo $x^{r}$. The comparison of the coefficients of the terms $x^{i} y^{d-i}$ for $1 \leq i \leq d$ implies that $F$ is a multiple of $x^{r}$ and $a_{i}=\mu^{i} u^{d-i} b_{i}$ for $2 \leq i \leq d$. Replacing $\mu u^{-1}$ by a new $\mu$, we obtain $a_{i}=\mu^{i} b_{i}$ for $\mu \in \mathbb{C}^{*}, 2 \leq i \leq d$.

Conversely, if $a_{i}=\mu^{i} b_{i}(2 \leq i \leq d)$ for $\mu \in \mathbb{C}^{*}$, then we can determine an isomorphism $\varphi$ by

$$
\begin{aligned}
\varphi(x)= & \mu x, \\
\varphi(y)= & y+x^{r} G(x), \\
\varphi(z)= & \mu^{-r}\left[z-x^{-r}\left\{\left(\varphi(y)^{d}+b_{2}(\mu x)^{2} \varphi(y)^{d-2}+\cdots+b_{d}(\mu x)^{d}\right)\right.\right. \\
& \left.\left.\quad-\left(y^{d}+a_{2} x^{2} y^{d-2}+\cdots+a_{d} x^{d}\right)\right\}\right],
\end{aligned}
$$

where $G(x) \in \mathbb{C}[x]$.

Let $\widetilde{X}(d, r)$ be the affine hypersurface in $\mathbb{A}^{3}$ defined by equation (2) in Lemma 2.6 which has the transition functions given in assertion (6) of the same lemma. Let $\widetilde{X^{\prime}}(d, s)$ be a similar affine hypersurface in $\mathbb{A}^{3}$ with the equation

$$
x^{s} z+\left(y^{d}+a_{1}^{\prime} x y^{d-1}+\cdots+a_{d-1}^{\prime} x^{d-1} y+a_{d}^{\prime} x^{d}\right)=1
$$

and the transition functions

$$
g_{\lambda \omega}^{\prime}:=\varphi_{\lambda}^{\prime-1} \circ \varphi_{\omega}^{\prime}: \mathbb{A}_{*}^{1} \times \mathbb{A}^{1} \rightarrow \mathbb{A}_{*}^{1} \times \mathbb{A}^{1}, \quad(x, c) \mapsto\left(x, c+\frac{p_{\omega}^{\prime}(x)-p_{\lambda}^{\prime}(x)}{x^{s}}\right) .
$$

As in [5], we define a 3 -dimensional affine variety $\widetilde{X}(d, r, s)$ by glueing together $d$-copies of the affine 3 -space $\{\omega\} \times \mathbb{A}^{3}(\omega \in H(d))$ by the following identification:

$$
\left(\omega, x, c_{1}, c_{2}\right) \sim\left(\lambda, x, c_{1}+\frac{p_{\omega}(x)-p_{\lambda}(x)}{x^{r}}, c_{2}+\frac{p_{\omega}^{\prime}(x)-p_{\lambda}^{\prime}(x)}{x^{s}}\right), \quad x \neq 0 .
$$

The projection $\left(\omega, x, c_{1}, c_{2}\right) \mapsto\left(\omega, x, c_{1}\right)$ yields a morphism $\pi_{1}: \tilde{X}(d, r, s) \rightarrow \widetilde{X}(d, r)$ which is a principal $G_{a}$-bundle over $\widetilde{X}(d, r)$ with $G_{a}$ acting naturally on the coordinate $c_{2}$. Similarly, the projection $\left(\omega, x, c_{1}, c_{2}\right) \mapsto\left(\omega, x, c_{2}\right)$ gives rise to a principal $G_{a}$-bundle $\pi_{2}: \widetilde{X}(d, r, s) \rightarrow \widetilde{X^{\prime}}(d, s)$ with $G_{a}$ acting naturally on the coordinate $c_{1}$. Since every principal $G_{a}$-bundle over an affine variety is trivial [20, it follows that

$$
\widetilde{X}(d, r) \times \mathbb{A}^{1} \cong \widetilde{X}(d, r, s) \cong \widetilde{X^{\prime}}(d, s) \times \mathbb{A}^{1} .
$$

Hence the surfaces $\tilde{X}(d, r)$ have the non-cancellation property. 
Theorem 2.8. Let $d \geq 2$ and let $r, s>d$. Let $\widetilde{X}(d, r)$ and $\widetilde{X^{\prime}}(d, s)$ be the affine hypersurfaces defined by the equations $x^{r} z+\left(y^{d}+a_{2} x^{2} y^{d-2}+\cdots+a_{d} x^{d}\right)=1$ and $x^{s} z+\left(y^{d}+a_{2}^{\prime} x^{2} y^{d-2}+\cdots+a_{d}^{\prime} x^{d}\right)=1$, respectively. Then the following assertions hold:

(1) For any $r$ and $s$,

$$
\widetilde{X}(d, r) \times \mathbb{A}^{1} \cong \widetilde{X^{\prime}}(d, s) \times \mathbb{A}^{1} .
$$

(2) The isomorphism $\widetilde{X}(d, r) \cong \widetilde{X^{\prime}}(d, s)$ holds if and only if $r=s$ and $a_{i}^{\prime}=\mu^{i} a_{i}$ for $\mu \in \mathbb{C}^{*}$ and $2 \leq i \leq d$.

At this point, we do not know whether the isomorphism $\widetilde{X}(d, r) \times \mathbb{A}^{1} \cong \widetilde{X^{\prime}}(d, s) \times$ $\mathbb{A}^{1}$ in Theorem 2.8 is $H(d)$-equivariant or not. We shall show that the isomorphism in Theorem 2.8(1) is in fact $H(d)$-equivariant in some cases. The $H(d)$-action specified in assertion (1) of Lemma 2.6 is lifted to $\widetilde{X}(d, r, s)$ on $\omega$-charts as follows so that $\pi_{1}$ and $\pi_{2}$ are $H(d)$-equivariant:

$$
\lambda \cdot\left(\omega, x, c_{1}, c_{2}\right)=\left(\lambda \omega, \lambda x, \lambda^{1-r} c_{1}, \lambda^{1-s} c_{2}\right) \quad \text { for } \lambda \in H(d) .
$$

We look for $H(d)$-equivariant sections of $\pi_{1}$ and $\pi_{2}$. A section of $\pi_{1}: \tilde{X}(d, r, s) \rightarrow$ $\widetilde{X}(d, r)$ is expressed on $\omega$-chart as

$$
\{\omega\} \times \mathbb{A}^{2} \rightarrow\{\omega\} \times \mathbb{A}^{3}, \quad(x, c) \mapsto\left(x, c, \sigma_{\omega}(x, c)\right) .
$$

Hence an $H(d)$-equivariant section of $\pi_{1}$ is a family of $\sigma_{\omega} \in \mathbb{C}[x, c](\omega \in H(d))$, which is compatible with glueing maps and $H(d)$-actions.

Lemma 2.9. For the principal $G_{a}$-bundle $\pi_{1}: \tilde{X}(d, r, s) \rightarrow \widetilde{X}(d, r)$, an $H(d)$ equivariant section is given by a family of polynomials $\sigma_{\omega} \in \mathbb{C}[x, c], \omega \in H(d)$, satisfying the following conditions:

(1) For all $\omega, \lambda \in H(d)$ and $(x, c) \in \mathbb{A}_{*}^{1} \times \mathbb{A}^{1}$,

$$
\sigma_{\omega}(x, c)+\frac{p_{\omega}^{\prime}(x)-p_{\lambda}^{\prime}(x)}{x^{s}}=\sigma_{\lambda}\left(x, c+\frac{p_{\omega}(x)-p_{\lambda}(x)}{x^{r}}\right) .
$$

(2) For any $\omega, \lambda \in H(d)$,

$$
\lambda^{1-s} \sigma_{\omega}(x, c)=\sigma_{\lambda \omega}\left(\lambda x, \lambda^{1-r} c\right) .
$$

We can use relation (2) in the above lemma to compute $\sigma_{\lambda}$ from $\sigma_{1}$ :

$$
\sigma_{\lambda}(x, c)=\lambda^{1-s} \sigma_{1}\left(\lambda^{-1} x, \lambda^{r-1} c\right) .
$$

In terms of the function $\sigma_{1}$, conditions (1) and (2) in Lemma 2.9 are reformulated as in the following result. The proof is essentially the same as in [5] if one takes into account relation (5)(iv) of Lemma 2.6.

Lemma 2.10. Given a polynomial $\sigma=\sigma_{1} \in \mathbb{C}[x, c]$, define polynomials $\left\{\sigma_{\lambda} \mid\right.$ $\lambda \in H(d)\}$ by equation (4) above. Then conditions (1) and (2) in Lemma 2.9 are satisfied if and only if $\sigma$ satisfies

$$
\lambda^{1-s} x^{s} \sigma\left(\lambda^{-1} x, \lambda^{r-1}\left(c+\frac{p_{1}(x)-p_{\lambda}(x)}{x^{r}}\right)\right)=x^{s} \sigma(x, c)+p_{1}^{\prime}(x)-p_{\lambda}^{\prime}(x)
$$

for all $\lambda \in H(d),(x, c) \in \mathbb{A}_{*}^{1} \times \mathbb{A}^{1}$. 
If there exists a polynomial $\sigma$ satisfying condition (5) in Lemma 2.10, then there is an $H(d)$-equivariant isomorphism

$$
\tilde{X}(d, r, s) \cong \tilde{X}(d, r) \times \mathbb{A}^{1}(1-s),
$$

where $\mathbb{A}^{1}(a)$ denotes the one-dimensional $H(d)$-module of weight $a$. In fact, an $H(d)$-equivariant isomorphism $\widetilde{X}(d, r) \times \mathbb{A}^{1}(1-s) \cong \widetilde{X}(d, r, s)$ is defined as follows on the $\omega$-chart for $\omega \in H(d)$ :

$$
\begin{aligned}
\left(\omega \times \mathbb{A}^{2}\right) \times \mathbb{A}^{1} & \rightarrow \omega \times \mathbb{A}^{3}, \\
\left(\left(\omega, x, c_{1}\right), c_{2}\right) & \mapsto\left(\omega, x, c_{1}, \sigma_{\omega}\left(x, c_{1}\right)+c_{2}\right) .
\end{aligned}
$$

Let $\tilde{V}(d, r)$ be the affine surface defined by $x^{r} z+y^{d}=1$. Then $\tilde{V}(d, r)$ is the universal covering of the affine pseudo-plane $V(d, r)$. The polynomial $p_{\omega}(x)$ corresponding to $\widetilde{V}(d, r)$ is $p_{\omega}(x)=\omega$. Let $\widetilde{V}(d, r, s)$ be the affine variety glueing $\{\omega\} \times \mathbb{A}^{3}$ for $\omega \in H(d)$ with transition functions of $\widetilde{V}(d, r)$ and $\widetilde{V}(d, s)$ just as we constructed $\widetilde{X}(d, r, s)$. In [5], it is shown that there exist $H(d)$-equivariant sections of $\widetilde{V}(d, r, s) \rightarrow \widetilde{V}(d, r)$ for any $r$ and $s$. The next result is a key fact in finding $H(d)$-equivariant sections, which is due to tom Dieck in the case $u=1$.

Proposition 2.11. Let $u$ and $t$ be positive integers and let $1 \leq u \leq d-1$.

(1) There exists a unique polynomial $Q_{u, t}(x) \in \mathbb{C}[x]$ satisfying the following properties:

(i) $Q_{u, t}(\lambda x)=\lambda^{u} Q_{u, t}(x)$ for any $\lambda \in H(d)$.

(ii) $\operatorname{deg} Q_{u, t}(x)=u+(t-1) d$.

(iii) $Q_{u, t}(1+x)-1$ is divisible by $x^{t}$.

(2) Let $P_{u, t}(x)$ be the polynomial defined by the equation $Q_{u, t}(1+x)-1=$ $x^{t} P_{u, t}(x)$. Then for any $\lambda \in H(d)$,

$$
\lambda^{-t}(x+1-\lambda)^{t} P_{u, t}\left(\lambda^{-1}(x+1-\lambda)\right)=\lambda^{-u}\left(x^{t} P_{u, t}(x)+1-\lambda^{u}\right) .
$$

Proof. (1) By the property (i) and (ii), $Q_{u, t}(x)$ is written as

$$
Q_{u, t}(x)=\sum_{j=0}^{t-1} a_{j} x^{u+j d} .
$$

By property (iii), the coefficients $a_{j}$ must satisfy linear equations. The determinant of the coefficient matrix of the system of the linear equations in $a_{0}, \ldots, a_{t-1}$ is

$$
\left|\begin{array}{cccc}
1 & 1 & \cdots & 1 \\
\left(\begin{array}{c}
u \\
1
\end{array}\right) & \left(\begin{array}{c}
u+d \\
1
\end{array}\right) & \cdots & \left(\begin{array}{c}
u+(t-1) d \\
1
\end{array}\right) \\
\cdots & \cdots & \cdots & \cdots \\
\left(\begin{array}{c}
u \\
t-1
\end{array}\right) & \left(\begin{array}{c}
u+d \\
t-1
\end{array}\right) & \cdots & \left(\begin{array}{c}
u+(t-1) d \\
t-1
\end{array}\right)
\end{array}\right|
$$

where the binomial coefficient $\left(\begin{array}{l}a \\ b\end{array}\right)$ for $a<b$ is defined to be zero. Note that $\left(\begin{array}{c}u+j d \\ i\end{array}\right)$ is a polynomial in $j d$ of degree $i$. By adding a linear combination of the first $i$ rows to the $(i+1)$-th row, the $(i+1)$-th row becomes $1 / i$ ! times of $\left(0, d^{i},(2 d)^{i}, \cdots\right.$, $\left.(t-1)^{i} d^{i}\right)$. Hence the determinant reduces to a non-zero multiple of the Vandermonde determinant and its value is non-zero. Thus we can determine the coefficients $a_{j}$, and the polynomial $Q_{u, t}(x)$ is uniquely determined. 
(2) By the definition of $P_{u, t}(x), Q_{u, t}(x)$ is written as

$$
Q_{u, t}(x)=1+(x-1)^{t} P_{u, t}(x-1) .
$$

Then the required relation follows from property (i) of $Q_{u, t}(x)$.

Theorem 2.12 (cf. tom Dieck [5]). Let $d \geq 2$ and $r, s \geq 1$. Then for any $r$ and $s$, there exists an $H(d)$-equivariant isomorphism

$$
\tilde{V}(d, r) \times \mathbb{A}^{1}(1-s) \cong \widetilde{V}(d, s) \times \mathbb{A}^{1}(1-r) .
$$

Proof. It suffices to find $\sigma(x, c)$ satisfying

$$
\lambda^{1-s} x^{s} \sigma\left(\lambda^{-1} x, \lambda^{r-1}\left(c+\frac{1-\lambda}{x^{r}}\right)\right)=x^{s} \sigma(x, c)+1-\lambda
$$

for all $\lambda \in H(d),(x, c) \in \mathbb{A}_{*}^{1} \times \mathbb{A}^{1}$. Let $a$ and $t$ be integers such that $a=-s+r t$, $t>0$ and $a \geq 0$. Set $\sigma(x, c)=x^{a} c^{t} P_{1, t}\left(x^{r} c\right)$, where $P_{1, t}(x)$ is the polynomial defined in Proposition 2.11(2). Then one easily verifies that $\sigma(x, c)$ satisfies the above condition, and the assertion follows.

Remark. There is a $\mathbb{C}^{*}$-action on $\widetilde{V}(d, r)$ defined by

$$
\mu \cdot(x, y, z)=\left(\mu x, y, \mu^{-r} z\right) \quad \text { for } \mu \in \mathbb{C}^{*},
$$

which is the lift-up of the $\mathbb{C}^{*}$-action on the affine pseudo-plane $V(d, r)$ (cf. Lemma 2.5 and the statement below it). One verifies that the isomorphism in Theorem 2.12 is in fact an $H(d) \times \mathbb{C}^{*}$-equivariant isomorphism

$$
\widetilde{V}(d, r) \times \mathbb{A}^{1}(1-s,-s) \cong \widetilde{V}(d, s) \times \mathbb{A}^{1}(1-r,-r),
$$

where $\mathbb{A}^{1}(a, b)$ denotes the one-dimensional $H(d) \times \mathbb{C}^{*}$-module with weight $a$ for $H(d)$ and with weight $b$ for $\mathbb{C}^{*}$.

In some cases, we can find a polynomial $\sigma$ satisfying the condition in Lemma 2.10 and write down $\sigma$ explicitly. First, we consider the case $r=s=2$.

Lemma 2.13. Let $\widetilde{X}(d, 2)$ and $\widetilde{X^{\prime}}(d, 2)$ be the affine surfaces defined by $x^{2} z+$ $f(x, y)=1$ and $x^{2} z+h(x, y)=1$, respectively, where $f(x, y)$ and $h(x, y)$ are monic homogeneous polynomials of degree $d$. Then for any $d \geq 2$ there is an $H(d)$ equivariant isomorphism

$$
\widetilde{X}(d, 2) \times \mathbb{A}^{1}(-1) \cong \widetilde{X^{\prime}}(d, 2) \times \mathbb{A}^{1}(-1) .
$$

Proof. Let $\widetilde{X}(d, 2,2)$ be the affine variety obtained by $\widetilde{X}(d, 2)$ and $\widetilde{X^{\prime}}(d, 2)$. For the principal $G_{a}$-bundle $\tilde{X}(d, 2,2) \rightarrow \widetilde{X}(d, 2), p_{1}(x)$ and $p_{1}^{\prime}(x)$ in Lemma 2.9 are both of the form $1+a x$ for $a \in \mathbb{C}$. Since $p_{\lambda}(x)=\lambda p_{1}\left(\lambda^{-1} x\right)$ and $p_{\lambda}^{\prime}(x)=\lambda p_{1}^{\prime}\left(\lambda^{-1} x\right)$, condition (5) in Lemma 2.10 is reduced to

$$
\lambda^{-1} x^{2} \sigma\left(\lambda^{-1} x, \lambda\left(c+\frac{1-\lambda}{x^{2}}\right)\right)=x^{2} \sigma(x, c)+1-\lambda
$$

for all $\lambda \in H(d),(x, c) \in \mathbb{A}_{*}^{1} \times \mathbb{A}^{1}$. Set $\sigma(x, c)=x^{d-1}+c$. Then $\sigma$ satisfies the above condition and it follows that $\widetilde{X}(d, 2,2) \cong \widetilde{X}(d, 2) \times \mathbb{A}^{1}(-1)$. Since $\sigma(x, c)$ gives rise to an $H(d)$-equivariant section of the principal $G_{a}$-bundle $\widetilde{X}(d, 2,2) \rightarrow \widetilde{X^{\prime}}(d, 2)$, we have the assertion.

Next, we consider the case where $\widetilde{X}(d, r)$ is defined by the equation $x^{r} z+y^{d}+$ $a x^{d}=1$ with $a \in \mathbb{C}$. 
Lemma 2.14. Let $d<r \leq 2 d$. Suppose that $\widetilde{X}(d, r)$ is defined by $x^{r} z+y^{d}+a x^{d}=1$ with $a \in \mathbb{C}$. Then there exists an $H(d)$-equivariant isomorphism

$$
\widetilde{X}(d, r) \times \mathbb{A}^{1}(1-r) \cong \widetilde{V}(d, r) \times \mathbb{A}^{1}(1-r) .
$$

Proof. Let $\tilde{X}(d, r, r)$ be the affine variety obtained by $\widetilde{X}(d, r)$ and $\widetilde{V}(d, r)$. The polynomial $\sigma$ which gives rise to an $H(d)$-equivariant section of the principal $G_{a^{-}}$ bundle $\widetilde{X}(d, r, r) \rightarrow \widetilde{X}(d, r)$ must satisfy condition (5) in Lemma 2.10 with $r=s$, $p_{\lambda}(x)=\lambda\left(1-(a / d) x^{d}\right)$ and $p_{\lambda}^{\prime}(x)=\lambda$. Define $\sigma(x, c) \in \mathbb{C}[x, c]$ by

$$
\sigma(x, c)=-\frac{a^{2}}{d^{2}} x^{2 d-r}+\left(1+\frac{a}{d} x^{d}\right) c .
$$

Then $\sigma$ satisfies the condition and we obtain an $H(d)$-equivariant isomorphism $\widetilde{X}(d, r, r) \cong \widetilde{X}(d, r) \times \mathbb{A}^{1}(1-r)$. Similarly, one easily verifies that

$$
\tau(x, c)=\left(1-\frac{a}{d} x^{d}\right) c
$$

satisfies

$$
\lambda^{1-r} x^{r} \tau\left(\lambda^{-1} x, \lambda^{r-1}\left(c+\frac{1-\lambda}{x^{r}}\right)\right)=x^{r} \tau(x, c)+p_{1}(x)-p_{\lambda}(x)
$$

and gives rise to an $H(d)$-equivariant section of $\tilde{X}(d, r, r) \rightarrow \widetilde{V}(d, r)$. Hence $\widetilde{X}(d, r, r) \cong \widetilde{V}(d, r) \times \mathbb{A}^{1}(1-r)$, and the assertion follows.

Combining Lemma 2.14 and Theorem 2.12, we obtain the following.

Lemma 2.15. Let $d<r \leq 2 d$ and $d<s \leq 2 d$. Suppose that $\widetilde{X}(d, r)$ and $\widetilde{X^{\prime}}(d, s)$ are defined by $x^{r} z+y^{d}+a x^{d}=1$ and $x^{s} z+y^{d}+a^{\prime} x^{d}=1$ with $a, a^{\prime} \in \mathbb{C}$, respectively. Then there exists an $H(d)$-equivariant isomorphism

$$
\widetilde{X}(d, r) \times \mathbb{A}^{1}(1-r) \times \mathbb{A}^{1}(1-s) \cong \widetilde{X^{\prime}}(d, s) \times \mathbb{A}^{1}(1-r) \times \mathbb{A}^{1}(1-s) .
$$

Now, resume the set-up in Lemmas 2.9 and 2.10 , and suppose that $r \equiv s \equiv 1$ $(\bmod d)$. Then we can find a polynomial $\sigma$ as satisfying the condition in Lemma 2.10 so that there exists an $H(d)$-equivariant isomorphism $\widetilde{X}(d, r) \times \mathbb{A}^{1} \cong \widetilde{X^{\prime}}(d, s) \times \mathbb{A}^{1}$, where $\mathbb{A}^{1}$ is the trivial $H(d)$-module.

Theorem 2.16. Let $d \geq 2$ and $r \equiv s \equiv 1(\bmod d)$. Then there is an $H(d)$ isomorphism

$$
\widetilde{X}(d, r) \times \mathbb{A}^{1} \cong \widetilde{X^{\prime}}(d, s) \times \mathbb{A}^{1} .
$$

Proof. We first show that $\widetilde{X}(d, r) \times \mathbb{A}^{1} \cong \widetilde{V}(d, 1) \times \mathbb{A}^{1}$ for any $r \equiv 1(\bmod d)$. Consider the affine variety $\widetilde{X}(d, r, 1)$ obtained by $\widetilde{X}(d, r)$ and $\widetilde{V}(d, 1)$. Then, the polynomial $\sigma$ which gives rise to an $H(d)$-equivariant section of the principal $G_{a^{-}}$ bundle $\widetilde{X}(d, r, 1) \rightarrow \widetilde{X}(d, r)$ must satisfy

$$
x \sigma\left(\lambda^{-1} x, c+\frac{p_{1}(x)-p_{\lambda}(x)}{x^{r}}\right)=x \sigma(x, c)+1-\lambda
$$

for all $\lambda \in H(d),(x, c) \in \mathbb{A}_{*}^{1} \times \mathbb{A}^{1}$, where $p_{1}(x)=1+a_{1} x+\cdots+a_{r-1} x^{r-1}$ with $a_{i} \in \mathbb{C}$. It follows from $p_{\lambda}(x)=\lambda p_{1}\left(\lambda^{-1} x\right)$ that

$$
\begin{aligned}
p_{1}(x)-p_{\lambda}(x) & =p_{1}(x)-\lambda p_{1}\left(\lambda^{-1} x\right) \\
& =(1-\lambda)+\sum_{i=2}^{k d} a_{i}\left(1-\lambda^{1-i}\right) x^{i},
\end{aligned}
$$


where $k$ is a non-negative integer such that $r=1+k d$. Set

$$
\sigma(x, c)=f_{0}(x)+f_{1}(x) c
$$

where

$$
f_{0}(x)=a_{2} x+\cdots+a_{k d} x^{k d-1}, \quad f_{1}(x)=x^{k d}
$$

Then $\sigma$ satisfies the above condition, and it follows that $\widetilde{X}(d, r, 1) \cong \widetilde{X}(d, r) \times \mathbb{A}^{1}$ as $H(d)$-varieties. Next, we find a polynomial $\tau$ which gives rise to an $H(d)$-equivariant section of the principal $G_{a}$-bundle $\tilde{X}(d, r, 1) \rightarrow \widetilde{V}(d, 1)$. The polynomial $\tau$ must satisfy

$$
x^{r} \tau\left(\lambda^{-1} x, c+\frac{1-\lambda}{x}\right)=x^{r} \tau(x, c)+p_{1}(x)-p_{\lambda}(x)
$$

for all $\lambda \in H(d),(x, c) \in \mathbb{A}_{*}^{1} \times \mathbb{A}^{1}$. Note that

$$
p_{1}(x)-p_{\lambda}(x)=(1-\lambda)+\sum_{\substack{2 \leq i \leq d \\ 0 \leq j \leq k-1}} a_{i+j d}\left(1-\lambda^{1-i}\right) x^{i+j d} .
$$

Set

$$
\tau(x, c)=c^{r} P_{1, r}(x c)+\sum_{\substack{2 \leq i \leq d \\ 0 \leq j \leq k-1}} a_{i+j d} c^{r-i-j d} P_{d-i+1, r-i-j d}(x c)
$$

where $P_{i, j}(x)$ is the polynomial defined in Proposition 2.11(2). Then $\tau$ satisfies the above equation, and an $H(d)$-equivariant isomorphism $\widetilde{X}(d, r, 1) \cong \widetilde{V}(d, 1) \times \mathbb{A}^{1}$ holds. Hence we obtain an $H(d)$-equivariant isomorphism $\widetilde{X}(d, r) \times \mathbb{A}^{1} \cong \widetilde{V}(d, 1) \times$ $\mathbb{A}^{1}$ for any $r \equiv 1(\bmod d)$. Since we have an $H(d)$-isomorphim $\widetilde{X^{\prime}}(d, s) \times \mathbb{A}^{1} \cong$ $\widetilde{V}(d, 1) \times \mathbb{A}^{1}$ for $s \equiv 1(\bmod d)$, there exists an $H(d)$-isomorphism $\tilde{X}(d, r) \times \mathbb{A}^{1} \cong$ $\widetilde{X^{\prime}}(d, s) \times \mathbb{A}^{1}$.

By Lemma 2.7 and Theorem 2.16, we obtain families of non-isomorphic affine surfaces $\widetilde{X}(d, r)$ with equivariant non-cancellation property. By taking the quotients by $H(d)$, we obtain families of affine pseudo-planes with non-cancellation property.

Theorem 2.17. Let $d \geq 2$ and let $r, s>1$ and $r \equiv s \equiv 1(\bmod d)$. Let $\widetilde{X}(d, r ; f)$ be the affine hypersurface defined by $x^{r} z+f(x, y)=1$, where $f(x, y)$ is of the form $y^{d}+a_{2} x^{2} y^{d-2}+\cdots+a_{d} x^{d}$ with $a_{j} \in \mathbb{C}$. Then the quotient of $\widetilde{X}(d, r ; f)$ by the Galois group $H(d)$ is an affine pseudo-plane $X(d, r ; f)$ of type $(d, r)$, and the following assertions hold:

(1) For any $r$ and $s$,

$$
X\left(d, r ; f_{1}\right) \times \mathbb{A}^{1} \cong X\left(d, s ; f_{2}\right) \times \mathbb{A}^{1},
$$

where $f_{1}$ and $f_{2}$ are monic homogeneous polynomials of the form stated above.

(2) The isomorphism $X\left(d, r ; f_{1}\right) \cong X\left(d, s ; f_{2}\right)$ holds if and only if $r=s$ and $f_{1}(x, y)=f_{2}(\mu x, y)$ for $\mu \in \mathbb{C}^{*}$. 


\section{An APPLiCATION}

Let $G$ be a reductive algebraic group. As an application of the results in the previous section, we construct the examples of families of affine $G$-varieties without equivariant cancellation property.

Let $Y=\operatorname{Spec} R$ be an affine $G$-variety such that the invariant subring $R^{G}$ is a polynomial ring $\mathbb{C}[x]$ for $x \in R^{G}$. Let $\tilde{Y}(d, r ; f)$ be a hypersurface in $Y \times \mathbb{A}^{2}=$ Spec $R[y, z]$ defined by $x^{r} z+f(x, y)=1$, where $f(x, y)$ is a monic homogeneous polynomial of degree $d$. Then $\tilde{Y}(d, r ; f)$ has a $G_{a}$-action induced by a locally nilpotent $R$-derivation $D=x^{r} \partial_{y}-f_{y} \partial_{z}$. Since the $G_{a}$-action commutes with the $G$-action, the inclusion $\operatorname{Ker} D=R \hookrightarrow R[y, z]$ induces a $G$-equivariant $\mathbb{A}^{1}$-fibration $\widetilde{Y}(d, r ; f) \rightarrow Y$. Let $\tilde{\pi}: \widetilde{Y}(d, r ; f) \rightarrow \widetilde{X}(d, r ; f)$ and $\pi: Y \rightarrow \mathbb{A}^{1}=\operatorname{Spec} \mathbb{C}[x]$ be the algebraic quotients by $G$, where $\tilde{X}(d, r ; f)$ is the affine hypersurface in $\mathbb{A}^{3}=$ Spec $\mathbb{C}[x, y, z]$ defined by $x^{r} z+f(x, y)=1$. Then it follows that $\tilde{Y}(d, r ; f)=$ $\widetilde{X}(d, r ; f) \times_{\operatorname{Spec}} \mathbb{C}[x] Y$.

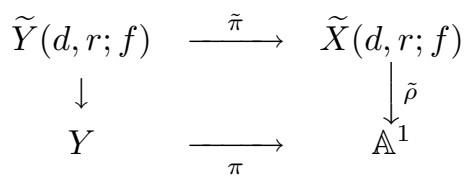

Theorem 3.1. Let $d \geq 2$ and let $r, s>d$. Let $\tilde{Y}\left(d, r ; f_{1}\right)$ and $\tilde{Y}\left(d, s ; f_{2}\right)$ be affine $G$-varieties defined by $f_{1}(x, y)$ and $f_{2}(x, y)$ as above, respectively, where $f_{1}$ and $f_{2}$ are homogeneous polynomials of the form $y^{d}+a_{2} x^{2} y^{d-2}+\cdots+a_{d} x^{d}$ for $a_{i} \in \mathbb{C}(2 \leq i \leq d)$. Then the following assertions hold:

(1) For any $r$ and $s$, there is a $G$-equivariant isomorphism

$$
\widetilde{Y}\left(d, r ; f_{1}\right) \times \mathbb{A}^{1} \cong \widetilde{Y}\left(d, s ; f_{2}\right) \times \mathbb{A}^{1} .
$$

(2) The isomorphism of $G$-varieties $\tilde{Y}\left(d, r ; f_{1}\right) \cong \widetilde{Y}\left(d, s ; f_{2}\right)$ holds if and only if $r=s$ and $f_{1}(x, y)=f_{2}(\mu x, y)$ for $\mu \in \mathbb{C}^{*}$.

Proof. The assertions follows from Theorem 2.8.

\section{REFERENCES}

[1] J. Ax, The elementary theory of finite fields, Ann. of Math. 88 (1968), 239-271. MR 0229613 $(37: 5187)$

[2] H. Bass and W. Haboush, Some equivariant K-theory of affine algebraic group actions, Comm. Algebra 15 (1987), 181-217. MR0876977 (88g:14013)

[3] J. Bertin, Pinceaux de droites et automorphismes des surfaces affines, J. Reine Angew. Math. 341 (1983), 32-53. MR0697306 (84f:14035)

[4] W. Danielewski, On the cancellation problem and automorphism group of affine algebraic varieties, preprint.

[5] T. tom Dieck, Homology planes without cancellation property, Arch. Math. (Basel) 59 (1992), 105-114. MR.1170634 (93i:14012)

[6] K. H. Fieseler, On complex affine surfaces with $\mathbb{C}^{+}$-action, Comment. Math. Helv. 69 (1994), 5-27. MR1259603 (95b:14027)

[7] T. Fujita, On the topology of non-complete algebraic surfaces, J. Fac. Sci. of the Univ. Tokyo, Ser. IA 29 (1982), 503-566. MR0687591 (84g:14035)

[8] R. V. Gurjar and M. Miyanishi, On a generalization of the Jacobian Conjecture, J. Reine Angew. Math. 516 (1999), 115-132. MR.1724617 (2001b:14094)

[9] R.V. Gurjar and M. Miyanishi, Automorphisms of affine surfaces with $\mathbb{A}^{1}$-fibrations, Michigan Math. J. 53 (2005), no. 1, 33-55. MR2125532 
[10] K. Masuda, Certain moduli of algebraic $G$-vector bundles over affine $G$-varieties, in Advanced Studies in Pure Mathematics 33, Math. Soc. of Japan, Tokyo (2002). MR.1890099 (2003a:14096)

[11] K. Masuda and M. Miyanishi, The additive group actions on $\mathbb{Q}$-homology planes, Ann. Inst. Fourier, Grenoble 53, 2 (2003), 429-464. MR1990003 (2004e:14073)

[12] K. Masuda and M. Miyanishi, Equivariant cancellation for algebraic varieties, Contemp. Math., 369, Amer. Math. Soc., Providence, RI, 2005. MR2126662

[13] M. Miyanishi, Lectures on curves on rational and unirational surfaces, Tata Institute of Fundamental Research, Springer, 1978. MR0546289 (81f:14001)

[14] M. Miyanishi, Open algebraic surfaces, Centre de Recherches Mathématiques, Vol. 12, Université de Montréal, Amer. Math. Soc., 2000. MR.1800276 (2002e:14101)

[15] M. Miyanishi, Singularities of normal affine surfaces containing cylinderlike open sets, J. Algebra 68 (1981), 268-275. MR0608535 (82k:14035)

[16] M. Miyanishi, Étale endomorphisms of algebraic varieties, Osaka J. Math. 22 (1985), 345-364. MR:0800978 (87f:14021)

[17] M. Miyanishi and K. Masuda, Generalized Jacobian Conjecture and Related Topics, Proceedings of the Internat. Conf. on Algebra, Arithmetic and Geometry, Tata Institute of Fundamental Research, 427-466, 2000. MR1940676 (2004a:14065)

[18] M. Miyanishi and T. Sugie, Homology planes with quotient singularities, J. Math. Kyoto Univ. 31 (1991), 755-788. MR1127098 (92g:14034)

[19] G. Schwarz, Exotic algebraic group actions, C. R. Acad. Sci. Paris 309 (1989), 89-94. MR1004947 (91b:14066)

[20] J.-P. Serre, Espaces fibrés algébriques, Séminaire C. Chevalley, Anneaux de Chow, Exposé 1, 1958.

[21] H. Sumihiro, Equivariant completion, J. Math. Kyoto Univ. 14 (1974), 1-28. MR0337963 $(49: 2732)$

[22] J. Wilkens, On the cancellation problem for surfaces, C. R. Acad. Sci. Paris 326 (1998), 1111-1116. MR 1647227 (99i:14040)

Mathematical Science II, Himeji Institute of Technology, 2167 Shosha, Himeji 6712201, JAPAN

E-mail address: kayo@sci.himeji-tech.ac.jp

School of Science \& Technology, Kwansei Gakuin University, 2-1 Gakuen, Sanda 669-1337, JAPAN

E-mail address: miyanisi@ksc.kwansei.ac.jp 